\title{
Students Corner-6
}

\author{
Mrutyunjaya Behera ${ }^{1}$ \\ ${ }^{1}$ Department of Cardiology, Visiting Consultant, Kalinga Hospital, \\ Bhubaneswar, Orissa, India \\ Ind J Car Dis Wom 2021;6:275-276.
}

\section{Q1: Accepted Mechanism of S3}

The genesis of early diastolic filling sound or third heart sound (S3) has remained controversial. There has been a lot of excellent clinical and investigative works that include experimental works in dogs to explain this auscultatory phenomenon. Many laboratory procedures, for example, apexcardiogram, accelerometry, frame by frame left ventricular (LV) geometric study during cardiac catheterization, combined echo-phonocardiographic recording, were utilized in the process. Some of the workable hypotheses, which were forwarded by different authors, are as follows:

1. One of the earliest physicians, Potain was the original investigator to study and ascertain the mechanism of S3 in 1880 . He attributed S3 to sudden cessation of distension of ventricle at the end of rapid filling phase (RFP) of cardiac cycle in early diastole. At the end of RFP ventricles quickly arrive at a point where fibrous resistance from its wall limits its distension and then abruptly stops, which produces a tension-a shock-the S3.

2. Ozawa et al in $1983^{1}$ in their both noninvasive (accelerometric) and invasive (LV angiographic study in catheterization laboratory which included frame by frame LV cavity analysis) works supported the concept that S3 was due to sudden intrinsic limitation of longitudinal expansion of LV wall during early diastolic filling, resulting in a negative jerk, that is, S3. This negative jerk was probably contributed by a tensing of intracardiac supporting structures that include papillary muscle, chordae tendineae, or by inherent limiting movement of myocardium itself.

3. Dock et al in $1955^{2}$ using hemodynamic data from LV and left atrium (LA) came up with the valvular theory as a mechanism of S3. They attributed S3 gallop to momentary closure of mitral valve (MV) in response to rapidly fluctu-
Address for correspondence Mrutyunjaya Behera, MD, DM, Department of Cardiology, Visiting Consultant, Kalinga Hospital, Bhubaneswar, Orissa, India (e-mail: mbehera2002@yahoo.com).

ating pressure difference in left-sided chambers. This theory was supported by Dayem and Raftery in $1966^{3}$ and Fleming in $1969^{4}$ because of the reasoning that S3 disappears in patients who have MV prosthesis. However, valvular theory is not an acceptable proposition as earlier improved studies using superior techniques by Kuo in $1957^{5}$ demonstrated that LA pressure frequently exceeded LV pressure at the time of S33, keeping the valve still open. Moreover, Coulshed and Epstein in $1972^{6}$ by their studies in cases of prosthetic valve replacement proved that MV valve apparatus is not necessary for the production of S3.

In summary, I still stick to the original idea of Potain. S3 occurs not during the RFP when there is a sudden rush of diastolic flow to the ventricle, but at the end of RFP, when transition occurs from RFP to slow filling phase and this sudden cessation of rapid filling leads to limitation of longitudinal expansion of LV wall that produces tension, a vibration, a shock, or a negative jerk that is S3. In simple words, S3 is the result of altered dynamics of LV relaxation during diastole, such that the ventricles vibrate on sudden cessation of early filling. This has been amply proved by many echo-phonocardiographic as well as clinicoangiographic studies. ${ }^{7-9}$

Q2: S3 in Mitral Regurgitation (MR) and Aortic Regurgitation (AR)

Apical S3 is very common finding in chronic MR as per reports of Bleiffer et al in $1960^{10}$ from their auscultatory and phonocardiographic studies. This was also an observation in the studies conducted by Nixon in $1961 .{ }^{11}$ Perloff and Harvey ${ }^{12}$ utilizing similar modes of noninvasive investigations in 1962 noted that S3 may be audible in both acute and chronic MR. However, this may not be audible in mild MR, but as the severity increases, it appears and becomes more prominent. A more recent veteran administration cooperative study in valvular heart disease by Folland and Krigel in $1992^{7}$ correlated
DOI https://doi.org/ 10.1055/s-0041-1742211. ISSN 2455-7854. (c) 2022. Women in Cardiology and Related Sciences. All rights reserved.

This is an open access article published by Thieme under the terms of the Creative Commons Attribution-NonDerivative-NonCommercial-License, permitting copying and reproduction so long as the original work is given appropriate credit. Contents may not be used for commercial purposes, or adapted, remixed, transformed or built upon. (https://creativecommons.org/ licenses/by-nc-nd/4.0/)

Thieme Medical and Scientific Publishers Pvt. Ltd., A-12, 2nd Floor, Sector 2, Noida-201301 UP, India 
S3 with angiographic grades of MR. A high prevalence of S3 to the tune of $46 \%$ was observed in MR. When the visual grade of MR (as assessed by angiography) was compared in patients with or without S3, a consistent, positive, and significant relation was demonstrated between increasing grades of MR and prevalence of S3. This relation was independent of LV ejection fraction (EF). Within each grade of MR, the prevalence of $\mathrm{S} 3$ was similar in patients with $\mathrm{EF}$ of $<50 \%$ and in those with $\mathrm{EF}>50 \%$. Their findings were consistent with results of experimental work of Ishimitsu et al in $1985^{13}$ in which S3 could be produced in dogs by either acute volume overload (MR) or myocardial dysfunction (ischemia).

To summarize, a S3 can be audible in majority cases of chronic severe MR as well as acute MR. S3 in former does not necessarily indicate presence of $L V$ dysfunction.

About S3 in AR: It is not a very common finding in chronic severe AR. Moreover, if it is present, it may not be audible because of prominent diastolic murmur in these cases. A clearly audible $\mathrm{S} 3$ would indicate starting of LV dysfunction in this subset of patients. In contrast, a loud S3 is very frequently heard in acute AR. In the excellent clinicoangiographic correlative analysis of Folland et $\mathrm{al}^{7}$ that involved nearly 358 cases of free AR, an increased prevalence of S3 was noted only in patients with severe AR. The prevalence of S3 was 13,20 , and $32 \%$ in mild, moderate, and severe AR, respectively. The data indicates that only one-third of cases of severe AR are likely to have an audible S3.

Q3: "AF- effect of Cycle Length on S2"

My comments are as follows-"There are hardly any references to the subject in literatures, neither there are any worthwhile comment in older clinical text books. Two aspects are considered when we discuss about S2. They are splitting and intensity of individual component in diseased states. My personal feeling is that in a person without valvular heart disease, pulmonary arterial hypertension, or shunt lesions or outflow obstruction, where there is no burden on the hangout interval S2 splitting and intensity will remain normal whatever is the cycle length of AF. In patients with right ventricular obstruction or RBBB there will be persistence of wide splitting because of basic hemodynamics in former and conduction delay in later. However the quantum of splitting may be a little less in shorter cycles regardless of phases of respiration. Similarly in patients with congenital heart disease like Atrial septal defect, wide and fixed splitting phenomenon will persist though magnitude of gap between the 2 components may be less in shorter cycles because of the reason that the hangout interval is partially flow dependent."

Same topic was discussed by Seepana and Vala in their article on second heart sound. ${ }^{14}$ According to the author, second heart sound splitting is reflection of differences between ventricles in terms of onset, duration of systole, and hangout interval. Any condition that selectively influences one ventricle like BBB, pressure/volume overload, and hangout interval will change A2-P2 interval. Longer cycles in AF affect both ventricles equally and thus no change in A2P2 interval unless it results in aberrant conduction like RBBB-unlikely in longer cycles (resulting in wide split) or change in hangout interval that is flow dependent.

Basically, in a lone AF patient without underling heart disease, AF cycle length does not have effect on S2 split or intensity. These remarks are my personal opinion. I cannot corroborate them with any concrete study or reference.

\section{Conflict of Interest}

None.

\section{References}

1 Ozawa Y, Smith D, Craige E. Origin of the third heart sound. II. Studies in human subjects. Circulation 1983;67(02):399-404

2 Dock W, Grandell F, Taubman F. The physiologic third heart sound: its mechanism and relation to protodiastolic gallop. Am Heart J 1955;50(03):449-464

3 Dayem MKA, Raftery EB. Mechanisms of production of heart sounds based on records of sounds after valve replacement. Am J Cardiol 1966;18(06):837-842

4 Fleming JS. Evidence for a mitral valve origin of the left ventricular third heart sound. Br Heart J 1969;31(02):192-199

5 Kuo PT. The gallop sounds. Studies of the mechanism of production. Circulation 1957;16:276

6 Coulshed N, Epstein EJ. Third heart sound after mitral valve replacement. Br Heart J 1972;34(03):301-308

7 Folland ED, Krigel BJ, et al. Implication of third heart sound in patients with valvular heart disease (In the Veteran Administration study on valvular heart disease). N Engl J Med 1992; 327:458-462

8 Van de Werf F, Minten J, Carmeliet P, De Geest H, Kesteloot H. The genesis of the third and fourth heart sounds. A pressure-flow study in dogs. J Clin Invest 1984;73(05):1400-1407

9 Van de Werf F, Boel A, Geboers J, et al. Diastolic properties of the left ventricle in normal adults and in patients with third heart sounds. Circulation 1984;69(06):1070-1078

10 Bleifer S, Dack S, Grishman A, Donoso E. The auscultatory and phonocardiographic findings in mitral regurgitation. Am J Cardiol 1960;5:836-842

11 Nixon PGF. Clinical and phonocardiographic study of S3 in majority cases of mitral regurgitation. Br Heart J 1961;23v(06): 677-689

12 Perloff JK, Harvey WP. Auscultatory and phonocardiographic manifestations of pure mitral regurgitation. Prog Cardiovasc Dis 1962;5:172-194

13 Ishimitsu T, Smith D, Berko B, Craige E. Origin of the third heart sound: comparison of ventricular wall dynamics in hyperdynamic and hypodynamic types. J Am Coll Cardiol 1985;5(2 Pt 1):268-272

14 Seepana L, Vala DR. Second heart sound. Ind J Car Dis Wom 2020; 5:76-86 